\title{
An Indigenous Cost Effective Design for Aerosol Prevention during Airway Manipulation
}

\author{
Virendra Jain ${ }^{1}$ Rana Patir ${ }^{2}$ Hari H. Dash ${ }^{1}$ \\ ${ }^{1}$ Department of Neuroanaesthesia, Fortis Memorial Research \\ Institute, Gurugram, Haryana, India \\ ${ }^{2}$ Department of Neurosurgery, Fortis Memorial Research Institute, \\ Gurugram, Haryana, India
}

J Neuroanaesthesiol Crit Care:2020;7:176-178

Aerosol contamination of operation theater personnel is a great concern during airway manipulation of patients with suspected or proven COVID-19. A plethora of devices such as intubation boxes and barrier devices have been recommended to minimize the spread of aerosols in environment. ${ }^{1-3}$ However, the available devices have some major constraints such as rigid frame, heavy, fixed in design with great restriction in hand mobility, and problem of sterilization. ${ }^{2}$ Keeping these points in mind, we have designed an indigenous wire mesh device for barrier protection during airway manipulation along with our senior neurosurgeon. The concept of a stable enclosure, light-weight, cost-effective design along with unrestricted natural movement of the hands and clear visibility was possible with the novel design. The final device was designed and fabricated by the second author.

This is a wired mesh design, in which steel wires are fashioned into a cage resembling a halfsphere. The device has more space at the bottom to accommodate the patient's head, upper torso and airway devices. The head rests on an additional loop of wire, which is swung below the half sphere, anchoring and stabilizing the flexible cage (-Fig. 1A). Two $\mathrm{C}$-arm polyurethane covers are applied in opposing directions over the frame (one from above and one from below) to make it an enclosed space. The edges of the $\mathrm{C}$-arm cover are tucked in between the two rows of steel wire, so that they do

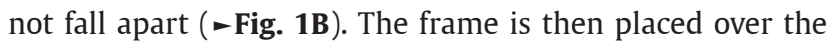
head and upper torso of the patient using appropriate cuts on the lower cover. For easy access of the anesthesiologist's hands, a triple layer of plastic membrane is stretched within a self-locking 3D-printed double hoop of co-polyester. The membranes are then cut in an opposing trifurcation (like a Mercedes Benz sign). This allows for free hand access while at the same time largely contains the inner atmosphere inside canopy, preventing aerosol egress. The plastic sheet design

\author{
Address for correspondence Hari H. Dash, MD, Department of \\ Neuroanaesthesia, Fortis Memorial Research Institute, Sector-44, \\ Gurugram, Haryana 122002, India \\ (e-mail: dr.harihardash@gmail.com).
}

allows for flexibility in the placement of the hand access rings as well as addition of more access points if required for second anesthesiologist and OT assistant ( - Fig. 1C). This frame is hanged from the angle bar fixed at the edge of the OT table, so that the height can be adjusted for different operators. It is also possible to maintain a negative pressure within the enclosure with a high-volume air extraction via suction.

The advantages of this design are a stable canopy enclosure with a clear and wide view for the anesthesiologist. The flexible wire frame and use of polyethylene covers allow better hand movement and more natural hand-eye coordination. Additionally, the space between the two hands can be adjusted. This system can be easily assembled, autoclaved, and is cost-effective and reusable. During use, negative pressure suction can be applied, which prevents fogging and enhances safety by removing infected aerosols. The height can be adjusted as per the anesthesiologist's requirement.

Limitations of this device can be the dispersal of contained aerosol inside the canopy. To prevent spread of aerosol during use, negative suction is applied inside the canopy, which removes aerosols. Even after the completion of the procedure, we recommend suctioning the enclosed canopy for at least 5 minutes to remove aerosol as much as possible. Also, the Mercedes Benz sign-shaped incision in the rings prevents egress of aerosols. The plastic sheets are disposable C-arm covers, which are routinely used in operation theaters. These are disposed of after use as per hospital biomedical waste protocols.

This frame takes around 5 minutes to assemble, which can be a disadvantage in emergency situations. We have been using this frame and all our colleagues have found it to be very useful. We would request more colleagues to use it, so that further improvement deemed necessary can be performed.
DOI https://doi.org/ 10.1055/s-0040-1715920 ISSN 2348-0548. (c) 2020. Indian Society of Neuroanaesthesiology and Critical Care. This is an open access article published by Thieme under the terms of the Creative Commons Attribution-NonDerivative-NonCommercial-License, permitting copying and reproduction so long as the original work is given appropriate credit. Contents may not be used for commercial purposes, or adapted, remixed, transformed or built upon. (https://creativecommons.org/licenses/by-nc-nd/4.0/). Thieme Medical and Scientific Publishers Pvt. Ltd., A-12, 2nd Floor, Sector 2, Noida-201301 UP, India 

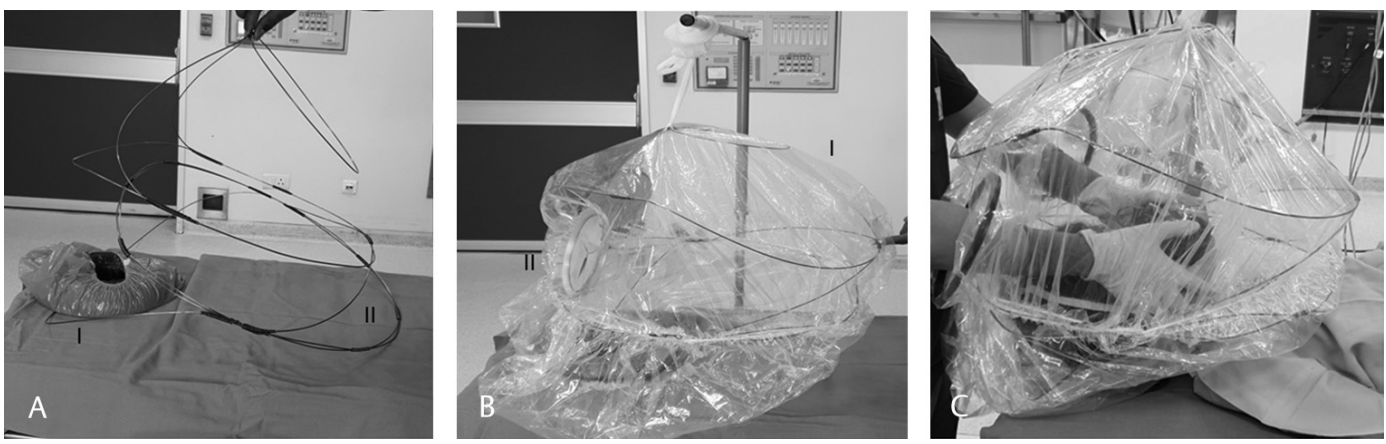

Fig. 1 (A) Wire mesh design for barrier device to reduce aerosol spread. I. Wire loop placed beneath head ring for stabilization. II. Two rows of steel wire. (B) Figure showing enclosed space in which C-arm plastic covers are placed from above and below to provide protection from all sides. I. Covers are tucked in between two rows of steel wire to prevent them from falling. II. Superimposed 3D printed co-polyester rings in which slits are made to create space for hands. (C) Figure showing hand placement while doing mask ventilation.

\section{Conflict of Interest}

None declared.

\section{References}

1 Canelli R, Connor CW, Gonzalez M, Nozari A, Ortega R. Barrier enclosure during endotracheal intubation. $\mathrm{N}$ Engl J Med 2020;382(20):1957-1958
2 Rosenblatt WH, Sherman JD. More on barrier enclosure during endotracheal intubation. N Engl J Med 2020;382(21):e69

3 Patwa A, Shah A, Garg R, et al. All India difficult airway association (AIDAA) consensus guidelines for airway management in the operating room during the COVID -19 pandemic. Indian J Anaesth 2020;64:107-115 